\title{
La práctica del silencio como una herramienta educativa: directrices para la educación basada en competencias ${ }^{1}$
}

\author{
Francisco José Lería Dulčić ${ }^{2}$ \\ Orcid: https://orcid.org/0000-0001-6326-2567
}

\section{Resumen}

Las características de los entornos educativos contemporáneos, abundantes en actividades y uso excesivo de las tecnologías digitales, alertan a la necesidad de proveer al estudiante de una experiencia de equilibrio psicofísico al interior del aula. Esto ha despertado el interés de los y las docentes por el silencio como un acto educativo crucial para un proceso formativo menos saturado de información y más harmónico en naturaleza. Así, el presente artículo revisa literatura científica sobre el concepto de silencio como un potenciador de la neurofisiología, por ejemplo, la neurogénesis y estado basal del cerebro; pero también el desarrollo psicosocial, las habilidades auto regulativas atencionales y la calidad del procesamiento del lenguaje, sugiriendo así que su práctica posee las condiciones necesarias para ser considerada como una herramienta pedagógica, tarea a la cual este artículo está dedicado. Se expone una breve revisión de la orientación contemplativa en la enseñanza y el aprendizaje, como aquel enfoque teórico y práctico interesado en el silencio como una estrategia de uso y alcance pedagógico, potenciadora del crecimiento psicológico y la transformación social, a través del desarrollo de la conciencia y la promoción del comportamiento ético-relacional. Se describen algunas implicaciones para la educación basada en competencias y la articulación de su práctica con el desarrollo de las competencias procedimentales. Finalmente, algunas limitaciones son abordadas para generar conciencia sobre el mejor camino para la promoción del silencio y sus beneficios para los entornos educativos.

\section{Palabras clave}

Educación basada en competencias - Educación alternativa - Orientación pedagógica Actualización de los conocimientos - Atención plena.

1- Se agradece a los estudios de postgrado científico interdisciplinario en Lenguaje y Neurociencia Cognitiva (JEKON-LCN), Universidad de Zagreb, por apoyar este trabajo y a Laertes Mariani por su colaboración en la traducción al castellano de este artículo.

2- Universidad de Atacama; Copiapó, Chile. Contacto: francisco.leria@uda.cl 


\title{
The practice of silence as an educational tool: guidelines for competence-based education
}

\begin{abstract}
The characteristics of contemporary educational environments, abundant in activities and often-excessive use of digital technologies, highlight the need for providing psychophysical balance to the student inner experience, current situation that stimulates teachers' interest to the consideration of silence as an educative act, crucial to any less saturated, and more balanced formative process. Current scientific literature is reviewed here about silence as an enhancer of neurophysiology, e.g.: on neurogenesis and default network inductor; and psychosocial development, e.g.: on self-regulative attentional skills and/or language processing quality; suggesting it already involves the preconditions to be systematically introduced as a pedagogical tool, a task still to be done and to which this paper tributes. For this goal, a brief review on the contemplative orientation in teaching and learning is exposed as a theoretical and practical framework interested in silence as a tool of learning, psychological growth, and social transformation, through awareness development and relational-ethical behavior. Some implications are described then for competence-based education, and the possibility of articulating the practice of silence with the development of procedural competences. Finally, some limitations are addressed in order to raise awareness of the best path to tread in promoting silence and its benefits for teaching and learning environments
\end{abstract}

\section{Keywords}

Competence-based education - Alternative education - Pedagogical guidance - Knowledge updating - Mindfulness.

\section{Introducción}

En la última década se ha observado un gran interés por el estudio del bienestar del estudiante a lo largo de su desarrollo evolutivo, lo cual ha promovido el surgimiento de variadas y novedosas ideas para su desarrollo (BARRY; CLARKE; DOWLING, 2017). La investigación específica en esta área ha destacado que el logro de la integración cuerpomente, así como la satisfacción con la vida, pueden ser facilitadas mediante el cultivo de un estado mental de quietud, tranquilidad y silencio (TRAN et al., 2020), ofreciendo al campo de la educación información valiosa respecto de los efectos neurofisiológicos y psicosociales implicados en tales estados psicofísicos, así como conjuntamente la metodología y técnicas necesarias para alcanzarlos. Esta línea de investigación se le ha a menudo denominado: la revolución silenciosa (ZAJONC, 2013); e involucra conceptos como la atención plena, el desarrollo atencional y la concienciación, pero simultáneamente las contribuciones de disciplinas científicas menos conocidas, como la neurociencia 
y la educación contemplativa y -en un sentido más amplio-, la espiritualidad. Estas disciplinas han sido la fuente de datos empíricos duros para el diseño de varios programas educativos destinados al desarrollo de las habilidades auto regulativas atencionales y comportamentales, a través de la práctica del silencio (HUTCHINSON; HUWS; DORJEE, 2018; LÓPEZ-HERNÁEZ, 2016; WATERS et al., 2014; MEIKLEJOHN et al., 2012); lo cual ha proporcionado una mirada panorámica a las proyecciones y aplicabilidad de esta evidencia empírica emergente para la contribución de un enfoque más holístico, que promueva el crecimiento y bienestar psicosocial del estudiante.

Sin embargo, existe otra cara en la comprensión del silencio y su utilización en los entornos educativos, que no se basa en los efectos positivos que ocurren cuando los y las estudiantes lo practican, ni en la cantidad de programas exitosos que puedan existir, sino en la equiparación e integración adecuada de los datos empíricos con los requerimientos actuales del currículo. En este sentido, los especialistas sostienen que los diferentes pasos en el desarrollo de un currículo han de articularse con cada nueva información que difiera del currículo previsto, y así lo pueda entonces debidamente enriquecer (SHUEY et al., 2019). Por lo tanto, y en sentido recíproco, los nuevos datos han de adecuarse al currículo para garantizar su gestión e inclusión desde un punto de vista pedagógico. Teniendo en mente lo anterior, el objetivo general del presente trabajo es contribuir a estas reflexiones en dos corrientes de análisis: por un lado, la consideración de la evidencia empírica como un eje fundamental para la construcción de estrategias de intervención efectivas para el desarrollo de la atención basada en las prácticas del silencio, y por otro, la propuesta de algunas ideas sobre el camino correcto para la equiparación más arriba mencionada entre el silencio, su teoría, práctica y los requisitos curriculares actuales, tal como se plantea en la educación basada en competencias (en adelante EBC).

\section{La necesidad de silencio}

La pedagogía a menudo se ha mantenido reservada en cuanto a la utilización sistemática de la experiencia contemplativa en cualquiera de sus formas, a pesar de la evidencia que sugiere que las habilidades auto regulativas pueden emerger a consecuencia de su práctica, con un reconocido impacto en el aprendizaje y el comportamiento (TONIOLOBARRIOS; BRASIL; PITT, 2020; DENG et al., 2019). La comunidad educativa se ha mostrado así parcialmente esquiva respecto de esta evidencia, principalmente debido a la noción de que los comportamientos no orientados a objetivos específicos y predeterminados, no poseen un papel relevante en el proyecto educativo, en contraste con los comportamientos funcionales y preferiblemente dirigidos a una meta, que para el caso del concepto de silencio, no constituiría una expresión educativa entendida como una expresión activa y agencial (HAO, 2011); con evidencias que confirman estas predisposiciones subjetivas del cuerpo docente (LERÍA DULČIĆ; SALGADO ROA; SASSO ORELLANA, 2018). Aunque esto puede ser entendido a la luz de la relevancia de las habilidades funcionales, tema expuesto en innumerables estudios que nutren en la actualidad los modelos educativos seculares, la necesidad de la experiencia de silencio no es un tema cliché para los tiempos educativos en los cuales se vive actualmente, y por los cuales los investigadores son llamados a explorar esta temática. 
La era de la sobreestimulación ha alcanzado proporciones inesperadas, aumentando constantemente sus efectos y poniendo en riesgo la salud psíquica y mental de las personas. Varios autores concuerdan en que las tecnologías del mundo moderno, especialmente las digitales: "[...] perpetúan ciertas formas de pensamiento creadas por ellas, pero una vez puestas en práctica, nuestro pensamiento y comportamiento son asimismo reformados" (HADAR; ERGAS, 2018, p. 1); y un número de estudios lo confirman posible de ser aquí citados. Por ejemplo, la exposición a programas televisivos caracterizados por cambios de imágenes rápidos durante los primeros tres años de vida está relacionado con déficit atencional e hiperactividad en el trascurso de la infancia (CHRISTAKIS et al., 2018); el uso de la tecnología digital a la hora de dormir correlaciona con una alta masa corporal (FULLER et al., 2017); solo el 5,2\% de los estudiantes declara abiertamente que el ruido en el aula les es agradable (CASTR0; MORALES, 2015); y también se observa una preocupante asociación entre el uso excesivo de las redes sociales y la densidad de la materia gris en las regiones del cerebro implicadas en la cognición social (VON DER HEIDE; VYAS; OLSON, 2014). A pesar de que los ejemplos mencionados anteriormente se pueden encontrar en la literatura científica actual, la relación dinámica entre sobreestimulación y silencio, aún no ha sido plenamente reconocida por los docentes y profesionales del campo educativo como una respuesta a esta problemática.

\section{¿Qué es practicar el silencio en un ambiente educativo?}

La comprensión del silencio generalmente se ha limitado a distintos referentes, principalmente desde una ausencia de ruido hasta una forma resistencia, disciplina, omisión, entre otros (WU; WENNING, 2016). Sin embargo, cuando el silencio es intencional, voluntario y mediado por la práctica, la experiencia del sujeto es expandida dando lugar a nuevas profundidades vivenciales para quien lo practica. Hoy en día, esto ha tomado la forma de la denominada conciencia plena y/o prácticas contemplativas, generalmente definidas como un ejercicio atencional cuerpo-mente, que puede ser practicado por el sujeto de manera individual o en grupos. Existen variados tipos de estas prácticas, algunas derivadas desde las tradiciones espirituales ancestrales del mundo, otras de reciente aparición, solo por mencionar aquí algunas: la atención plena; la meditación en posición sentada y al caminar; la meditación guiada para la exploración corporal; la atención auditiva consciente; el movimiento consciente; el arte en muchas de sus formas; la alimentación consciente; y muchas otras más (ROBINSON; EID, 2017). Aunque todas estas prácticas en sí incluyen al silencio, muchas otras actividades también poseen la característica de generar un modo especial de atención y sensibilidad corporal (DAHL; DAVIDSON, 2018), por ejemplo, la interpretación musical; el debate; la peregrinación; el compromiso y acción social; los rituales; y varias otras (The Tree of Contemplative Practices, 2000). Una práctica contemplativa clásica y estandarizada generalmente involucra algunas preparaciones e inducciones verbales (ejemplo: relajación progresiva; respiración secuencial; cotejo corporal; fantasías guiadas; movimientos sincrónicos; y otros); la mantención de la sensación o el estado alcanzados $\mathrm{y}$, en algunos casos, la expresión de la experiencia (verbalmente, a través del arte o acciones expresivas similares). En los entornos escolares a menudo las prácticas contemplativas se realizan en grupos 
de forma diaria o semanal, pero con una mayor frecuencia como parte de las actividades programadas en espacios y un tiempo previamente definido (LERÍA DULĆIĆ; SALGADO ROA; SASSO ORELLANA, 2019).

Entre los enfoques relacionados con los propósitos recién descritos, la educación contemplativa ha intentado sistemática y consistentemente promover la práctica del silencio y desarrollo de la atención en los entornos educativos (WEARE, 2019; WATERS et al., 2014). Varios contextos han hecho posible su reaparición en occidente (LERÍA DULĆIĆ, 2017), y se define como una forma de educación holística en la que las prácticas contemplativas se integran en los entornos educativos para potenciar el aprendizaje y el desarrollo psicosocial. Se nutre desde las contribuciones de varias áreas científicas para el enriquecimiento de la práctica educativa con procedimientos científicamente validados con un carácter preventivo universal y orientado a la mejora del desarrollo global del estudiante.

\section{Los hallazgos científicos y el impacto multidimensional de las prácticas de silencio}

La investigación empírica asociada al silencio ha abarcado diferentes dimensiones constitutivas del individuo, con un corpus de evidencia en rápido crecimiento que aborda prácticas de silencio reconocidas, como la atención plena, el yoga y/o la meditación budista (COOPER; YAP; BATALHA, 2018; SHUTE, 2018; ZENNER; HERRNLEBEN; WALACH, 2014). Este artículo se relaciona con varias líneas en la literatura científica que enfatizan el amplio impacto de las prácticas de silencio de especial interés para fines educativos, junto a la exposición de algunas líneas de investigación menos conocidas en el área.

\section{La neurogénesis y el efecto de los estados inducidos de reposo en el cerebro}

La experiencia de silencio ha mostrado jugar un papel relevante cuando se trata del desarrollo encefálico. Un estudio ejemplar y clásico en este sentido -El sonido del silencio es música para el corazón (LARSEN, 2005)- describe el efecto del silencio sobre la frecuencia respiratoria, cardíaca y la presión arterial, las cuales disminuyen en los sujetos experimentales, por debajo de los niveles basales previos. De este modo, se ha concluido que los estados inducidos de silencio y descanso contribuyen al equilibrio homeostático del organismo, fundamentales después de la actividad continuada, así como previo a la acción, confirmada por variados estudios en el campo educativo. Por ejemplo, el entrenamiento de relajación a través de las técnicas de bio retroalimentación ayuda a aliviar las perturbaciones emocionales antes de los exámenes en estudiantes universitarios (TAHSINI et al., 2017), y las prácticas de atención plena disminuyen el estrés y la ansiedad académica (BAMBER; KRAENZLE, 2016). Sin embargo, el impacto del silencio es más expansivo de lo que inicialmente fue considerado, donde otro sugestivo estudio ha contribuido a su visualización. El artículo “¿El silencio es oro?” (KIRSTE et al., 2013) tiene como objetivo exponer los efectos de los estímulos auditivos en la neurogénesis del hipocampo, principal región encefálica asociada a la memoria y el aprendizaje. La disminución de todas las condiciones experimentales (como las llamadas sin previo aviso, 
música y silencio), excepto el ruido blanco, da como resultado una mayor proliferación celular incluso después de siete días. Aunque muchos de estos estudios se han realizado en sujetos experimentales, otros trabajos científicos han relacionado los estados de reposo cerebral inducidos por el silencio en practicantes de meditación, mostrando también mayor densidad neuronal del hipocampo (LUDERS; THOMPSON; KURTH, 2015) y, por ejemplo, menos errores cometidos en tareas de aprendizaje memorístico (GREENBERG et al., 2018).

Como se concluye más arriba, la ausencia de sobreestimulación es una condición vital para la restauración biológica y, en consecuencia, relevante de ser considerada por los y las profesionales en el campo educativo que busquen las mejores condiciones psicofisiológicas para el aprendizaje. En este sentido, el silencio debe abordarse desde una perspectiva estructural, por ejemplo, considerando los estándares mínimos de aislamiento auditivo; así como desde una perspectiva funcional, es decir, desde el fomento de las experiencias educativas que garanticen la presencia de una cantidad adecuada de silencio durante el transcurso de un día habitual de aprendizaje.

\section{El estado basal del cerebro}

Tras haber ya enfatizado la importancia del silencio en la neurogénesis, es importante enfocar la atención en otro tema estrechamente relacionado: el tipo de red neuronal que es activada y la subsecuente capacidad emergente del cerebro para modular el rendimiento cognitivo y la experiencia emocional. El estado basal del cerebro o red neuronal por defecto (RND) es definida como un grupo mayor de regiones encefálicas interactuantes y altamente correlacionadas entre sí. Los hallazgos de la investigación han sugerido que la práctica del silencio está asociada con este tipo de conectividad funcional intersectorial en las regiones fronto-insulares y fronto-estratiales, cruciales en la regulación de los procesos atencionales top-down (arriba/abajo) y la regulación emocional (HERNÁNDEZ et al., 2018). Es particularmente interesante como el aprendizaje es un fenómeno complejo que requiere de flexibilidad para transformar las redes neuronales existentes en otras nuevas y remodeladas, proceso asociado con estas redes dinámicas de la RND. Es distintivo entonces de este tipo de conectividad encefálica el fomento de las condiciones óptimas para el aprendizaje. La evidencia sugiere que la RND está asociada con una mayor actividad metabólica en algunas áreas centrales del cerebro, las cuales albergan capacidades como la atención orientada al exterior (GARTENSCHLÄGER et al., 2017), concluyendo así que el desarrollo de las habilidades atencionales genera este tipo de conectividad que por defecto es más duradera, como es justamente logrado mediante las prácticas de conciencia plena y/o mindfulness (BAUER et al., 2019; MARUSAK et al., 2018).

\section{La domesticación del reflejo atencional}

La necesidad de nuestro cerebro de dirigir la atención a las constantes novedades y cambios en el medio ambiente ha generado una tecnología educativa enfocada a tales entornos de aprendizaje, variados en contenido y estimulación. Es conocido que el enriquecimiento ambiental afecta el sistema nervioso central a nivel funcional, anatómico y molecular, especialmente durante el período de la infancia (SALE, 2018). Sin 
embargo, una característica propia de nuestra época, este enriquecimiento es fácilmente transformado en sobreestimulación, obligando al cerebro a permanentemente lidiar con una multitud de estímulos, adaptándose a ellos y modelando la percepción del entorno percibido dentro de los límites de los datos procesados. Así, se ha descrito a la habilidad atencional como un proceso modulativo y dinámico que posee una influencia directa en los niveles de excitación biológica (DE BARBAR0; CLACKSON; WASS, 2016) y cuando esta habilidad es permanentemente perturbada o sobre estimulada, se esperan dificultades, así como desajustes conductuales (SCARPA, 2015). Esto deja a los y las docentes con una conclusión previamente conocida: la excitación fisiológica y cognitiva afecta a la capacidad de los niños y niñas para prestar atención, concentrarse y finalmente aprender. No obstante, un importante y útil dato empírico está ausente desde la perspectiva arriba enunciada: el cerebro codifica algunos aspectos en silencio de la misma manera que durante la estimulación física (TAMAKOSHI et al., 2016), sugiriendo, por lo tanto, que las habilidades atencionales logradas al practicar el silencio son codificadas de manera similar en el cerebro, pero con niveles de excitación más bajos y -por lo tanto- con menos interferencia para el posterior procesamiento cognitivo (MAK et al., 2017).

\section{La codificación de la percepción del tiempo}

Una de las dificultades habituales en el aula refiere a la característica impaciencia y prisa de los y las estudiantes, especialmente los más jóvenes, por terminar las tareas dadas lo más rápido posible. La curiosa particularidad de percibir que el tiempo transcurre más lento frente a una tarea más extensa, poco atractiva o dificultosa, generalmente provoca inquietud, distracción e insatisfacción, por lo tanto, se convierte en un deber diario para los y las docentes el aplicar estrategias que disminuyan tal efecto. Los investigadores en neurociencia cognitiva han dado a conocer significativos avances en la comprensión de como el cerebro da lugar a la percepción del sentido del tiempo, revelando así sorprendentes conocimientos respecto de la organización de las redes neuronales asociadas y como -efectivamente- su transmisión es físicamente más lenta en niños que en adultos (COSTELLO et al., 2009). La investigación neurocientífica sobre esta relación ha sugerido que la región medio-frontal del sistema límbico y otras zonas posteriores del cerebro median las funciones atencionales responsables de la percepción del tiempo, pero en el camino hacia la madurez, la percepción y discriminación temporal pasa progresivamente a estar bajo el control de las regiones focales, laterales fronto-estriatales y parietales del encéfalo. En otras palabras, la percepción del tiempo migra de una base sensorio/ emocional a una cognitivo/ejecutiva, y - en consecuencia la precisión cognitiva que de esto emerge implica una percepción del tiempo más prolongada: por ejemplo ver más detalles en una tarea implica que el tiempo es percibido como más lento (PÜTZ et al., 2012); y lo contrario ocurre cuando se perciben menos detalles y el tiempo se experimenta transcurriendo más rápido. Algunos estudios han descrito que las prácticas regulares de silencio facilitan el desarrollo de la capacidad de regulación de la atención en estados cotidianos no meditativos, con un impacto en la percepción del tiempo (WITTMANN; SCHMIDT, 2014), ayudando a percibirlo como ocurriendo más lentamente debido a la 
precisión de la percepción del intervalo de tiempo, pero sin la ansiedad característica de una percepción temporal más acelerada.

\section{El lenguaje de las emociones y el bienestar}

Los contextos semánticos provistos por el lenguaje tienen un impacto en las experiencias del sujeto a un nivel no solo psicológico explicativo, sino también neurofisiológico. La excitación del aparato fisiológico influye incluso en el uso especifico del lenguaje, particularmente en la categorización y la velocidad de las palabras habladas, especificamente aquellas con connotación negativa. Un buen ejemplo se observa en los estudios que han descrito como las palabras que expresan dolor físico y social provocan sentimientos más intensos y desagradables con tiempos de respuesta verbal más rápidos y una mayor excitación fisiológica (BORELLI et al., 2018). También se ha sugerido que las palabras de baja excitación fisiológica son cognitivamente categorizadas más rápido después de la relajación corporal, lo cual sugiere su disminución promueve el reconocimiento lingüístico de ciertos estímulos verbales y coincidentes con el estado de actual del sujeto (KEVER; GRYNBERG; VERMEULEN, 2017).

Estudios recientes han mostrado que el tipo de práctica de silencio y las palabras que son utilizadas en sus instrucciones, como por ejemplo en las diversas formas de meditación, modulan la forma en que los practicantes se refieren en el presente a sus sensaciones corporales, sentimientos y pensamientos, así como también a las formas de más tarde relatar sus experiencias (PRZYREMBEL; SINGER, 2018). Estos hallazgos sugieren que el tipo de práctica de silencio conduce a un cierto uso especifico de lenguaje y - por lo tanto - de redes semánticas específicas, que conllevan una modulación y gestión exitosa de la excitación neurofisiológica y cognitivo-afectiva. La importancia de esta evidencia empírica ha sido asociada al efecto inconsciente del uso de palabras relacionadas con las emociones, por ejemplo, cuando los y las docentes inducen en sus estudiantes estados de excitación psicofísica similares a los propios, cuando es posible predecir su agotamiento laboral a través de los niveles de cortisol matinales de sus estudiantes (OBERLE; SCHONERTREICHL, 2016). Siguiendo la investigación citada de Przyrembel y Singer (2018), las palabras relacionadas con las prácticas de silencio pueden -por lo tanto- contribuir a la modulación de una conciencia y disposición cuerpo-mente más equilibrada en contextos distintos a aquellos en los cuales tales prácticas han tomado lugar.

Estudios recientes han mostrado que el tipo de práctica de silencio y las palabras que son utilizadas en sus instrucciones, como por ejemplo en las diversas formas de meditación, modulan la forma en que los practicantes se refieren en el presente a sus sensaciones corporales, sentimientos y pensamientos, así como también a las formas de más tarde relatar sus experiencias (PRZYREMBEL; SINGER, 2018). Estos hallazgos sugieren que el tipo de práctica de silencio conduce a un cierto uso especifico de lenguaje y - por lo tanto - de redes semánticas específicas, que conllevan una modulación y gestión exitosa de la excitación neurofisiológica y cognitivo-afectiva. La importancia de esta evidencia empírica ha sido asociada al efecto inconsciente del uso de palabras relacionadas con las emociones, por ejemplo, cuando los y las docentes inducen en sus estudiantes estados de excitación psicofísica similares a los propios, cuando es posible predecir su agotamiento 
laboral a través de los niveles de cortisol matinales de sus estudiantes (OBERLE; SCHONERTREICHL, 2016). Siguiendo la investigación citada de Przyrembel y Singer (2018), las palabras relacionadas con las prácticas de silencio pueden -por lo tanto- contribuir a la modulación de una conciencia y disposición cuerpo-mente más equilibrada en contextos distintos a aquellos en los cuales tales prácticas han tomado lugar.

\section{El comportamiento prosocial y el silencio}

Existe una sólida línea de investigación respecto del silencio y las variables de orden socioemocional de particular interés para los y las docentes, la cual ha demostrado que las prácticas contemplativas impactan positivamente en el comportamiento prosocial (LUBERTO et al., 2017), especialmente en niños y niñas con rasgos manifiestos de hiperactividad (VIGLAS; PERLMAN, 2017), enriqueciendo la conectividad social (KOK; SINGER, 2017), los comportamientos empáticos (LANERI et al., 2017), y las conductas cooperativas (MONTERO-MARÍN et al., 2016). Sin embargo, expertos en el área han señalado que, debido a razones de naturaleza metodológica, existen algunas limitaciones a esta evidencia que han de ser consideradas (KREPLIN; FARIAS; BRAZIL, 2018).

\section{Directrices para la inclusión de prácticas de silencio en EBC}

Uno de los elementos importantes a considerar frente al resurgimiento del enfoque contemplativo en la educación occidental, refiere a su articulación técnica con el currículo vigente en sus muchas formas, lo que implica que los beneficios de las prácticas de silencio no son suficientes por sí solas para constituirse en motivo de interés y aceptación por parte de los profesionales del campo educativo. Por esa razón es necesario vincular la evidencia empírica con los requisitos curriculares actuales para de este modo, proporcionar un propósito educativo reconocible a las prácticas contemplativas de silencio. Se deben -por lo tanto- abordar una serie de aspectos relevantes que facilitar la consecución de este objetivo y así promover este enfoque junto a los estándares pedagógicos y elementos didácticos correspondientes.

\section{Un punto ciego en la EBC}

La EBC se ha constituido en el nuevo eje de la educación moderna, a veces con un crecimiento acrítico y exponencial (HUMPHREYS; CRINO; WILSON, 2017). Ha surgido desde un movimiento en las pedagogías seculares cuyo objetivo es enfatizar la funcionalidad y el rendimiento $y$, para ese objetivo, la necesidad de un conjunto creciente de capacidades interrelacionadas para hacer frente a las tareas profesionales y problemas de la vida real, denominadas competencias. Los cambios actuales en los enfoques educativos, así como en la innovación curricular, han intentado así alcanzar una comprensión más integral de qué tipo de competencias son relevantes de ser incluidas en este modelo educativo. Si bien habilidades tales como el aprendizaje autónomo, colaborativo, cooperativo y/o situado se han redefinido en su importancia e incluido activamente en el aula, también es posible introducir otro tipo de habilidades 
bajo la condición de que respondan adecuadamente a criterios de validez y consistencia teórica al interior del modelo.

En el marco del Espacio Europeo de Educación Superior (2009 apud PEGALAJAR PALOMINO, 2018); diferentes tipos de competencias han sido incluidas. Aunque la mayoría posee un carácter explícitamente funcional y referente a comportamientos observables mediante los cuales el sujeto hace frente a su entorno, otras competencias se caracterizan en su definición conceptual y procedimental, por una naturaleza menos funcional y centrada en el hacer. Un ejemplo se encuentra en las competencias interpersonales e intrapersonales, que no son exclusivamente instrumentales, sino que implican la comunicación de sentimientos, así como la cooperación e interacción social (ZERMEÑO; LOZANO-RODRÍGUEZ, 2016). A pesar de que este último tipo de competencias da algunas luces respecto de una experiencia más subjetiva que puede ser manejada por el sujeto, igualmente apunta funcionalmente a otro objeto, situación, entorno, persona o grupos, sin necesariamente fortalecer, hacer más consciente o más clara la comunicación interna e íntima del individuo consigo mismo. Desde esta perspectiva, una parte crucial de la experiencia humana es soslayada, que -sin embargo- puede ser considerada como una habilidad y/o competencia a pesar de las dificultades que enfrenta para ser incluida en la EBC debido a su naturaleza amplia y ausente de una definición operacionalmente clara que le permita transformarse en una acción educativa. Así, existen varios intentos de incluir un sentido más amplio de lo que se entiende bajo el concepto de competencia, por ejemplo, varios modelos de la inteligencia emocional y social poseen una o dos habilidades entendidas como desde adentro, referidas comúnmente como competencias de autoconciencia (OBERLE; SCHONERT-REICHL, 2017). Otros trabajos, como el de Leclerc y Horan (2017), han sugerido con un mayor grado de exactitud ocho competencias básicas que incluye una expansión de lo que se entiende por una habilidad y/o competencia, admitiendo una relación activa de la persona consigo misma. Para este caso, la comunicación interna/ externa es definida como:

[...] la capacidad de transmitir/intercambiar, de manera clara, efectiva y natural, pensamientos, sentimientos e información, tanto dentro de uno mismo como entre uno mismo y el mundo; escuchar, hablar y escribir con empatía; atraer a otros a través del lenguaje y las acciones; experimentar la alegría de la comunión [...]. (2017, p. 6).

La definición anterior implica una cualidad esencial de la experiencia subjetiva, una relación más activa y consciente del sujeto consigo mismo o funcionalidad subjetiva que puede considerarse como una competencia en el campo de las estrategias de autoconciencia y auto regulación (LEYLAND; ROWSE; EMERSON, 2019). En el campo educativo la consideración de este tipo de competencias es justificada debido la evidencia que muestra su asociación con el éxito académico (ORIOL et al., 2017), así como su impacto en el desarrollo de muchas otras habilidades cognitivas y conductuales (DENG et al., 2019). 


\section{Prácticas de silencio, dimensiones curriculares y cómo abordarlas}

Una de las clasificaciones más amplias y perteneciente a la EBC propone un modelo básico que permite vincular la teoría y la técnica pedagógica tras las prácticas de silencio con los requisitos curriculares actuales, denominado KDB (Know, Do, and Be; Saber, Saber hacer y Saber ser); términos que aclaran las dimensiones que deben incluirse en cualquier modelo basado en competencias (DRAKE; REID, 2018). Las dos primeras categorías no son problemáticas de identificar (por ejemplo: para competencias de Saber, se incluyen el manejo del contenido, pensamiento crítico y/o innovación; y las competencias del Saber hacer, como la gestión de información, la resolución de problemas y/o la colaboración). No obstante, hasta cierto punto el Saber ser no es igualmente fácil de identificar y por lo tanto de definir operacionalmente. Esta última dimensión se ha tradicionalmente caracterizado por abarcar políticas educativas que se centran en áreas como la salud mental, el crecimiento personal, el aprendizaje socioemocional, el cultivo de valores y/o el desarrollo actitudinal (BIALIK et al., 2015), no obstante, la EBC requiere también de su abordaje con ad hoc habilidades y prácticas educativas aplicables en el aula. Es importante notar aquí en favor del modelo tratado, que un currículo no reductivo de cualquier tipo debe incluir asimismo al menos tres factores, a los que les deben corresponder actividades educativas transversales que aborden al estudiante con una comprensión total de lo que significa la educación y el crecimiento personal. Por ejemplo, un currículo orientado solo al dominio cognitivo carecería de un sentido más amplio y, por lo tanto, no facilitaría el conocimiento de otras áreas (factor holístico), privaría de una experiencia significativa y trascendente más allá del elemento puramente racional (factor transformador), e impediría una experiencia global unitaria, ecológica e integral del estudiante consigo mismo y su medio ambiente (factor de integración) (FERRER; SHERMAN, 2009).

La EBC requiere de experiencias de aprendizaje que brinden un proceso formativo que preferiblemente incluya todas estas dimensiones y/o factores, generando prácticas didácticas adecuadas y aptas para su aplicación y desarrollo. En consecuencia, el silencio apunta a una significativa pero no exclusiva forma de abordar al dominio del Saber ser, mediante prácticas que no solo involucran el desarrollo de las habilidades metacognitivas, sino que también promueven la generación de experiencias socioemocionales significativas.

\section{La traducción del lenguaje teórico y técnico a la pedagogía}

La promoción de las prácticas de silencio debe en cierta medida responder a las dimensiones y factores más arriba descritos, enfocándose en la transformación de sus construcciones teóricas y técnicas de procedimiento a un lenguaje pedagógico adecuado para los y las docentes. Aunque consideramos que la educación para enriquecerse a sí misma debe nutrirse de otros campos de conocimiento, esto no siempre funciona debido a las interferencias propias en la traducción entre los distintos lenguajes disciplinarios. Al respecto, existen algunas ideas que han mostrado, por ejemplo, que la validación del enfoque contemplativo 
y sus prácticas pueden ocurrir al interior de las comunidades educativas aprovechando la neurociencia, conocida y aceptada por los y las docentes (ERGAS et al., 2018).

\section{La metacognición y el papel de las prácticas de silencio para su desarrollo}

En el contexto de estas reflexiones, es particularmente importante conocer cuáles son el tipo de habilidades de aprendizaje que se necesitan para el futuro. Las directrices de la Organización Cultural de las Naciones Unidas han ofrecido una visión para los próximos años respecto de qué priorizar en educación, planteando la pregunta: ¿Qué tipo de pedagogías son adecuadas para el siglo XXI? De esto han surgido varias propuestas, desde la renovación del enfoque curricular hasta la redefinición de los roles y función docente (SCOTT, 2015). Dentro de estas directrices se ha recomendado enérgicamente que la pedagogía actual debe centrarse explícitamente en la enseñanza de las habilidades metacognitivas y/o de segundo orden. Así, teniendo esta declaración en mente, queda claro que el desarrollo de las habilidades relacionadas con el hacer en contextos reales de trabajo es preferida y esperada; lo cual ampliamente abarca la importancia de enseñar habilidades de autogestión del comportamiento, pero también trabajar con los y las estudiantes en el manejo de sí mismos en un sentido más personal e individual. Atendiendo a la evidencia hasta ahora acumulada en la literatura en el área que sugiere el cultivo de las prácticas de silencio -como la atención plena- conducen al fortalecimiento del desarrollo de la metacognición (BAUER et al., 2019; COOPER; YAP; BATALHA, 2018; MAK et al., 2017; ZENNER; HERRNLEBEN; WALACH, 2014), se hace más fácil el considerar a estas prácticas como una estrategia pedagógica válida a ser incorporada en la EBC para el desarrollo de tales habilidades.

\section{Epistemologias actuales y su predilección y/o antagonismo por las prácticas de silencio}

Otro tema relevante para traer a la discusión se relaciona a la epistemología. Sus particulares influencias actuales en el campo educativo, de naturaleza secular, tendencia funcional y crítica, definen el significado final de la educación de tal manera, que el tipo de currículo aceptable no permitirá fácilmente incorporar otras posibilidades. Esta situación ocurre en parte debido a la fuerte influencia de las contingencias socioculturales que en la actualidad la sociedad experimenta. En lo que concierne a la epistemología educativa, a menudo se manifiesta en un continuo de dos extremos. Por un lado, el liberalismo ha privilegiado a la racionalidad y a una pedagogía funcional, visible en el orden globalizado y, por otro lado, la pedagogía crítica que ha argumentado este enfoque normativo promueve reflexiones sobre el yo y no sobre el sistema en el que el mismo se encuentra (HARTLEY, 2018). Con respecto a ambas orientaciones, es preferible pensar en un enfoque más propiamente holístico y no per se contradictorio, donde cada epistemología y los modelos educativos que se les atribuyen puedan sacar provecho de algunos de los beneficios de las prácticas de silencio, sin socavar sus nociones fundamentales. Una opción es lo que algunos autores prefieren considerar en relación a los enfoques interesados en el estudio de la conciencia, como lo es el concepto de Alfabetización noética (PAYMAL, 
2014), que se caracteriza por el objetivo de educar a los estudiantes acerca de la conciencia y las experiencias que tienen de sí mismos (ERGAS, 2015). Esto implica un enfoque que puede contribuir a la educación de una manera no invasiva, con una sólida evidencia empírica y orientado a desarrollar la conciencia de variadas formas, de las cuales cualquier epistemología educativa puede obtener beneficio.

\section{Tendencias disciplinarias y su impacto en la innovación curricular}

Es especialmente importante la consideración de una serie de condiciones para garantizar que una propuesta de tal relevancia como lo es la inclusión de las prácticas de silencio en el contexto educativo, no solo sea una moda disciplinaria, que espera a ser reemplazada por otras actividades más novedosas o llamativas. A menudo las propuestas que no provienen directamente desde el interior del campo educativo, experimentan un proceso con dos fases. En primer lugar, se da paso a un interés inicial y fase de entusiasmo que induce a considerar que la propuesta en cuestión es la solución para todos los problemas educativos que el sistema experimenta, un ejemplo típico es la traducción prematura de la neurociencia para fines educativos (THOMAS; ANSARI; KNOWLAND, 2019). Una segunda fase surge cuando el colectivo de profesionales se acostumbra y entonces busca un enfoque más fresco y novedoso. Si bien estas tendencias pueden concebirse como parte del desarrollo esperado para cada disciplina aplicada, al mismo tiempo puede constituirse en un impedimento para extraer las mejores contribuciones que las disciplinas científicas pueden ofrecer. Otro buen ejemplo de este riesgo se observa cuando el interés por un campo disciplinar aplicado es más bien dirigido por el entusiasmo, que la racionalidad y la evidencia empírica, hoy en cierta medida visible en el SEL (Socio-Emotional Learning o Aprendizaje socioemocional). Esta tendencia descubre una excesiva subjetivación de la experiencia formativa (MENÉNDEZ ÁLVAREZ-HEVIA, 2018); en desmedro de otras habilidades, por ejemplo, aquellas cognitivas de carácter de auto regulativo en naturaleza.

Lo anterior puede en parte corregirse respondiendo con más atención a los requisitos específicos del currículo contemporáneo, y un buen ejemplo de ello se encuentra en la neuro-didáctica (FEILER; STABIO, 2018), disciplina que transforma la evidencia de la neurociencia en estrategias pedagógicas, pero a su vez no es una parte constitutiva de los fundamentos del plan de estudios. La misma situación puede ser aplicable al concepto de silencio: lejos de la euforia investigativa de la cual es actualmente objeto, es posible ser incorporado al currículo, por ejemplo, enfatizando el desarrollo de las competencias socioafectivas, o como ya se ha señalado más arriba, el desarrollo de la metacognición. Así, el conocimiento empírico y el conocimiento disciplinario podrán alinearse adecuadamente.

\section{Estándares metodológicos para las prácticas de silencio}

La apreciación del silencio para fines educativos implica su uso en entornos de aprendizaje situados y reales y, para ello, se deben cumplir con los necesarios estándares metodológicos de calidad (SEMPLE; DROUTMAN; REID, 2016). Por ejemplo, se sugiere elegir estrictamente intervenciones respaldadas por la evidencia empírica, y del mismo 
modo definir el grado de falsabilidad de los programas a implementar. Estos estándares de calidad garantizan no solo la seriedad de la intervención, sino que también permiten la retroalimentación de información confiable para la validación de las prácticas de silencio en grupos específicos de sujetos y/o de sus comunidades educativas.

\section{Prácticas de silencio y posibilidades de tiempo para ser incorporadas en los entornos educativos}

Finalmente, el último, pero no menos importante tema a considerar a partir de la literatura disponible, destaca la posibilidad real de incorporar prácticas de silencio en los ambientes de aprendizaje actuales, frecuentemente llenos de actividades. La extensión del tiempo en las escuelas ha sido objeto de amplio debate público. En los Estados Unidos, Europa y varios países latinoamericanos se han llevado a cabo reformas que implican aumentar la jornada escolar (MARTINIC; VILLALTA, 2015). En la actualidad los niños pasan muchas horas en la escuela, llevando la punta Costa Rica, Colombia y Chile con niños y niñas asistiendo a jornadas de clases de entre más de 1000 a 1200 horas por año (OCDE, 2018). Es difícil pensar que una institución educativa pueda otorgar tiempo para la práctica del silencio por la práctica en sí misma. Aunque este es un gran problema socioeconómico que supera los límites de este artículo, tal vez exista una parcial solución en las experiencias de otros programas que han logrado exitosamente incorporar este tipo de prácticas de silencio en los entornos escolares. Son buenos ejemplos (casi todos en su denominación original en inglés): Stress Reduction and Mindfulness Curriculum, Mindful Moment, Still Quiet Place, Resilient Kids, Mindful Schools, Inner Explorer, Mindfulness in Schools Project [MiSP], Mindfulness curriculum for 11-18 year old.b, The School-Based Meditation Model, Growing with Mindfulness o El Rito del Silencio (LERÍA DULČIĆ; SALGADO ROA; SASSO ORELLANA, 2019). Todos estos programas, con sus fortalezas y debilidades, muestran la importancia de un enfoque multidimensional y ofrecen una serie de actividades conjuntas, como charlas introductorias, experiencias contemplativas, guias de planificación individual y grupal, lecturas teóricas, supervisión virtual y muchas otras actividades, que garantizan un espacio para la participación, difusión y validación del programa. Además, existen sugerencias sobre la importancia de la práctica permanente a lo largo del tiempo (LÓPEZHERNÁEZ, 2016), así como lo han comprendido varios de los programas recién mencionados.

\section{Conclusiones: un camino aún por recorrer}

En este artículo ha sido explorada la evidencia empírica y algunas ideas sobre las prácticas de silencio como una estrategia educativa valiosa y, por lo tanto, relevante de ser considerada en la EBC y, en general, en cualquier plan de estudios contemporáneo. Cada una de las ideas aquí revisadas puede ser objeto de un análisis detallado, motivo por el cual este artículo pretende constituirse en una guía para investigadores, creadores de políticas educativas y profesionales del campo. El reciente aumento del interés por la relación entre las prácticas de silencio y su impacto en el desarrollo cognitivo y socioafectivo ha llevado a la unificación de los datos empíricos con la práctica, así como a considerar algunas reflexiones respecto de lo que el proyecto educativo puede abarcar en un sentido 
inclusivo y más completo. Así como es entendida aquí, la noción del silencio, sus prácticas y los datos que de su estudio emergen, aluden a un pluralismo de dimensiones, de las cuales el sistema educativo puede extraer sus beneficios, sin necesariamente compartir todos sus valores y supuestos paradigmáticos centrales. Se destaca así la búsqueda por un enfoque curricular más equilibrado, especialmente en la EBC, la cual tiende muchas veces a minimizar un importante tipo de experiencia humana de central interés para el enfoque contemplativo: la habilidad de relacionarse profundamente con uno mismo, mediante las prácticas de silencio como una de sus estrategias centrales.

Existe acuerdo de que los sistemas educativos deben estar constituidos por propuestas que sean una proyección de desarrollo para la sociedad desde la cual emergen y que, así, contribuyan al crecimiento del cuerpo de conocimiento que la misma dispone. En este contexto, tal perspectiva abre el camino para que el concepto de silencio sea incorporado y utilizado sistemáticamente en los entornos de aprendizaje, basado en la evidencia empírica y con una validada aplicabilidad práctica. El objetivo final de este documento es despertar el interés en esta importante experiencia humana no solo basado en su impacto, sino también en la simple idea de que un lugar tranquilo en la dinámica educativa habitual puede converger en una nueva forma de experimentar el proyecto educativo en sí mismo, propio y un poco más cercano al clásico Conócete a ti mismo.

\section{Referencias}

BAMBER, Mandy; KRAENZLE, Joanne. Mindfulness-based meditation to decrease stress and anxiety in college students: A narrative synthesis of the research. Educational Research Review, Netherlands, v. 18, p. 1-32, 2016.

BARRY, Margaret; CLARKE, Aleisha; DOWLING, Katherine. Promoting social and emotional well-being in schools. Health Education, Sydney, v. 117, n. 5, p. 434-451, 2017.

BAUER, Clemens et al. From state-to-trait meditation: reconfiguration of central executive and default mode networks. eNeuro, Washington, DC, v. 6, n. 6, p. 1-17, 2019.

BIALIK, Maya et al. Character education for the $21^{\text {st }}$ century: what should students learn? Boston: Center for Curriculum Redesign, 2015.

BORELLI, Eleonora et al. The psycholinguistic and affective structure of words conveying pain. PLoS ONE, Rome, v. 13, n. 6, p. 1-29, 2018.

CASTRO, Marianella; MORALES, María Esther. Los ambientes de aula que promueven el aprendizaje, desde la perspectiva de los niños y niñas escolares. Educare, Heredia, v. 19, n. 3, p. 1-32, 2015.

CHRISTAKIS, Dimitri et al. How early media exposure may affect cognitive function: a review of results from observations in humans and experiments in mice. Proceedings of the National Academy of Sciences, Washington, DC, v. 115, n. 40, p. 9851-9858, 2018.

COOPER, David; YAP, Keong; BATALHA, Luisa. Mindfulness-based interventions and their effects on emotional clarity: a systematic review and meta-analysis. Journal of Affective Disorders, Netherlands, v. 235, p. 265-276, 2018. 
COSTELLO, Patricia et al. Semantic and subword priming during binocular suppression. Consciousness and Cognition, EEUU, v. 18, n. 2, p. 375-382, 2009.

DAHL, Cortland J.; DAVIDSON, Richard J. Mindfulness and the contemplative life: pathways to connection, insight, and purpose. Current Opinion in Psychology, United Kingdom, v. 28, p. 60-64, 2018.

DE BARBARO, Kaya; CLACKSON, Kaill; WASS, Sam. Infant Attention Is dynamically modulated with changing arousal levels. Child Development, EEUU, v. 88, n. 2, p. 629-639, 2016.

DENG, Yuqin et al. The role of mindfulness and self-control in the relationship between mind-wandering and metacognition. Personality and Individual Differences, United Kingdom, v. 141, p. 51-56, 2019.

DRAKE, Susan Margaret; REID, Joanne. Integrated curriculum as an effective way to teach $21^{\text {st }}$ century capabilities. Asia Pacific Journal of Educational Research, Singapore, v. 1, n. 1, p. 31-50, 2018.

ERGAS, Oren. The post-secular rhetoric of contemplative practice in the curriculum. In: WEXLER, Philip; HOTAM, Yotam. New social foundations for education: education in post-secular society. New York: Peter Lang, 2015. p. 107-130.

ERGAS, Oren et al. Contemplative neuroscience as a gateway to mindfulness: findings from an educationally framed teacher learning program. Mindfulness, New York, v. 9, n. 6, p. 1723-1735, 2018.

FEILER, Jacob; STABIO, Maureen. Three pillars of educational neuroscience from three decades of literature. Trends in Neuroscience and Education, Alemania, v. 13, p. 17-25, 2018.

FERRER, Jorge; SHERMAN, Jacob. The participatory turn: spirituality, mysticism, religious studies. Albany: State University of New York Press, 2009.

FULLER, Caitlyn et al. Bedtime use of technology and associated sleep problems in children. Global Pediatric Health, Los Angeles, v. 4, p. 1-8, 2017.

GARTENSCHLÄGER, Martin et al. Resting brain activity related to dispositional mindfulness: a PET study. Mindfulness, New York, v. 8, n. 4, p. 1009-1017, 2017.

GREENBERG, Jonathan et al. Reduced interference in working memory following mindfulness training is associated with increases in hippocampal volume. Brain Imaging and Behaviour, New York, v. 13, n. 2, p. 366-376, 2018.

HADAR, Linor; ERGAS, Oren. Cultivating mindfulness through technology in higher education: a Buberian perspective. Al \& SOCIETY, Alemania, p. 1-9, 2018.

HAO, Richie Neil. Rethinking critical pedagogy: implications on silence and silent bodies. Text and Performance Quarterly, United Kingdom, v. 31, n. 3, p. 267-284, 2011.

HARTLEY, David. The emergence of blissful thinking in the management of education. British Journal of Educational Studies, United Kingdom, v. 67, n. 2, p. 201-216, 2018.

HERNÁNDEZ, Sergio Elías et al. Gray matter and functional connectivity in anterior cingulate cortex are associated with the state of mental silence during sahaja yoga meditation. Neuroscience, Netherlands, v. 371, p. 395-406, 2018. 
HUMPHREYS, Leanne; CRINO, Rocco; WILSON, lan. The competences movement: origins, limitations, and future directions: the competences movement. Clinical Psychologist, EEUU, v. 22, n. 3, p. 290-299, 2017.

HUTCHINSON, Julia; HUWS, Jaci; DORJEE, Dusana. Exploring experiences of children in applying a schoolbased mindfulness programme to their lives. Journal of Child and Family Studies, New York, v. 27, n. 12, p. 3935-3951, 2018.

KEVER, Anne; GRYNBERG, Delphine; VERMEULEN, Nicolas. Congruent bodily arousal promotes the constructive recognition of emotional words. Consciousness and Cognition, EEUU, v. 53, p. 81-88, 2017.

KIRSTE, Imke et al. Is silence golden? Effects of auditory stimuli and their absence on adult hippocampal neurogenesis. Brain Structure and Function, Alemania, v. 220, n. 2, p. 1221-1228, 2013.

KOK, Bethany; SINGER, Tania. Effects of contemplative dyads on engagement and perceived social connectedness over 9 months of mental training: a randomized clinical trial. JAMA Psychiatry, EEUU, v. 74, n. 2, p. 126-134, 2017.

KREPLIN, Ute; FARÍAS, Miguel; BRAZIL, Inti. The limited prosocial effects of meditation: a systematic review and meta-analysis. Sci Rep, United Kingdom, v. 8, p. 2403, 2018.

LANERI, Davide et al. Mindfulness meditation regulates anterior insula activity during empathy for social pain. Human Brain Mapping, EEUU, v. 38, n. 8, p. 4034-4046, 2017.

LARSEN, Peter. The sound of silence is music to the heart. Heart, United Kingdom, v. 92, n. 4, p. 433-434, 2005.

LECLERC, Remi; HORAN, Roy. "Fit" for change: measuring designer competence. International Journal of Design Creativity and Innovation, United Kingdom, v. 6, n. 3-4, p. 185-210, 2017.

LERÍA DULČIĆ, Francisco José. Incorporation of the contemplative orientation in the educational practice of the $21^{\text {st }}$ century. Reice, Madrid, v. 15, n. 4, p. 67-85, 2017.

LERÍA DULČIĆ, Francisco José. Strengthening initial professional education in preschool teachers: contemplative program "The Rite of Silence". Aulas de Encuentro, Jaén, v. 21, n. 1, p. 1-16, 2019. (in press).

LERÍA DULČIĆ, Francisco José; SALGADO ROA, Jorge Alejandro; SASSO ORELLANA, Patricia Ester. Sentido subjetivo de educadoras de párvulos en el uso e impacto del silencio en el aprendizaje. Reice, Madrid, v. 16, n. 2, p. 77-97, 2018.

LEYLAND, Anna; ROWSE, Georgina; EMERSON, Lisa-Marie. Experimental effects of mindfulness inductions on self-regulation: systematic review and meta-analysis. Emotion, Sheffield, v. 19, n. 1, p. 108-122, 2019.

LÓPEZ-HERNÁEZ, Lara. Mindfulness techniques in the schools. Academic and personal development of its participants. REOP, Madrid, v. 27, n. 1, p. 134-146, 2016.

LUBERTO, Christina et al. A systematic review and meta-analysis of the effects of meditation on empathy, compassion, and prosocial behaviors. Mindfulness, New York, v. 9, n. 3, p. 708-724, 2017.

LUDERS, Eileen; THOMPSON, Paul; KURTH, Florian. Larger hippocampal dimensions in meditation practitioners: differential effects in women and men. Frontiers in Psychology, Lausanne, v. 6, n. 186, p. 1-6, 2015. 
MAK, Catherine et al. Efficacy of mindfulness-based interventions for attention and executive function in children and adolescents: a systematic review. Mindfulness, New York, v. 9, n. 1, p. 59-78, 2017.

MARTINIĆ, Sergio; VILLALTA, Marco. La gestión del tiempo en la sala de clases y los rendimientos escolares en escuelas con jornada completa en Chile. Perfiles Educativos, Ciudad de México, v. 37, n. 147, p. 28-49, 2015.

MARUSAK, Hilary et al. Mindfulness and dynamic functional neural connectivity in children and adolescents. Behavioural Brain Research, Netherlands, v. 336, p. 211-218, 2018.

MEIKLEJOHN, John et al. Integrating mindfulness training into K-12 education: fostering the resilience of teachers and students. Mindfulness, New York, v. 3, p. 291-307, 2012.

MEISTER, Hartmut et al. Verbal response times as a potential indicator of cognitive load during conventional speech audiometry with matrix sentences. Trends in Hearing, v. 22, n. 233121651879325, p. 1-11, 2018.

MENÉNDEZ ÁLVAREZ-HEVIA, David. Critical approach to emotional intelligence as a dominant discourse in the field of education. Revista Española de Pedagogía, Madrid, v. 76, n. 269, p. 7-23, 2018.

MONTERO-MARÍN, Jesús et al. Psychological effects of a 1-month meditation retreat on experienced meditators: the role of non-attachment. Frontiers in Psychology, Lausanne, v. 7, n. 1935, p. 1-11, 2016.

OBERLE, Eva; SCHONERT-REICHL, Kimberly. Stress contagion in the classroom? The link between classroom teacher burnout and morning cortisol in elementary school students. Social Science \& Medicine, United Kingdom, v. 159, p. 30-37, 2016.

OBERLE, Eva; SCHONERT-REICHL, Kimberly. Social and emotional learning: recent research and practical strategies for promoting children's social and emotional competence in schools. In: MATSON, Johnny. Handbook of social behavior and skills in children. Cham: Springer, 2017. p. 175-198.

OECD - Organisation for Economic Co-operation and Development. Teaching hours. [S. I.], OECD, 2018. Available from: https://data.oecd.org/teachers/teaching-hours.htm. Access in: 7 Jan. 2019.

ORIOL, Xavier et al. The role of self-control and grit in domains of school success in students of primary and secondary school. Frontiers in Psychology, Lausanne, v. 8, p. 1-9, 2017.

PAYMAL, Noemi. Pedagooogía 3000: una pedagogía para el tercer milenio. La Paz: Ox La-Hun, 2014.

PEGALAJAR PALOMINO, María del Carmen. Formación en competencias en alumnado universitario mediante prácticas basadas en aprendizaje cooperativo. Revista Complutense de Educación, Madrid, v. 29 , n. 3, p. 35-52, 2018.

PRZYREMBEL, Marisa; SINGER, Tania. Experiencing meditation: evidence for differential effects of three contemplative mental practices in micro-phenomenological interviews. Consciousness and Cognition, EEUU, v. 62, p. 82-101, 2018.

PÜTZ, Peter et al. Duration discrimination in the context of age, sex, and cognition. Journal of Cognitive Psychology, United Kingdom, v. 24, n. 8, p. 893-900, 2012.

ROBINSON, Michael; EID, Michael. The happy mind: cognitive contributions to well-being. Cham: Springer, 2017.

SALE, Alessandro. Systematic look at environmental modulation and its impact in brain development. Trends in Neurosciences, Cambridge, v. 41, n. 1, p. 4-17, 2018. 
SCARPA, Angela. Physiological arousal and its dysregulation in child maladjustment. Current Directions in Psychological Science, Washington, DC, v. 24, n. 5, p. 345-351, 2015.

SCOTT, Cynthia L. The futures of learning 3: what kind of pedagogies for the $21^{\text {st }}$ century? Paris: Unesco Education Research and Foresight, 2015. Available from: https://unesdoc.unesco.org/ark:/48223/ pf0000243126. Access on: 13 Mar. 2019.

SEMPLE, Randye; DROUTMAN, Vita; REID, Brittany Ann. Mindfulness goes to school: things learned (so far) from research and real-world experiences. Psychology in the Schools, Muncie, v. 54, n. 1, p. 29-52, 2016.

SHUEY, Elizabeth et al. "Curriculum alignment and progression between early childhood education and care and primary school: A brief review and case studies". OECD Education Working Papers, Paris, v. 193, 9. 1-93, 2019.

SHUTE, Rosalyn. Schools, mindfulness, and metacognition: a view from developmental psychology. International Journal of School \& Educational Psychology, Amsterdam, v. 7, n. 1, p. 1-14, 2018.

TAHSINI, Gholami Z. et al. Biofeedback-aided relaxation training helps emotional disturbances in undergraduate students before examination. Applied Psychophysiology and Biofeedback, EEUU, v. 42, n. 4, p. 299-307, 2017.

TAMAKOSHI, Seiji et al. Entire sound representations are time-compressed in sensory memory: evidence from MMN. Frontiers in Neuroscience, Netherlands, v. 10, n. 347, p. 1-7, 2016.

THE TREE of contemplative practices. [S. I.], 15 July 2000. Available from: http://www.contemplativemind. org/practices/tree. Access on: 20 Dec. 2018.

THOMAS, Michael; ANSARI, Daniel; KNOWLAND, Victoria. Annual research review: educational neuroscience: progress and prospects. Journal of Child Psychology and Psychiatry, United Kingdom, v. 60, n. 4, p. 477-492, 2019.

TONIOLO-BARRIOS, Mariana; BRASIL, Alexander; PITT, Leyland, F. Nine prolific years: an analysis of publications in mindfulness. Mindfulness, Switzerland, v. 11, n. 3, p. 1-13, 2020.

TRAN, Bach Xuan et al. Global mapping of interventions to improve quality of life using mind-body therapies during 1990-2018. Complementary Therapies in Medicine, Churchill Livingstone, v. 49, n. 102350, p. 1-8, 2020.

VIGLAS, Melanie; PERLMAN, Michal. Effects of a mindfulness-based program on young children's self-regulation, prosocial behaviour and hyperactivity. Journal of Child and Family Studies, EEUU, v. 27, n. 4, p. 1150-1161, 2017.

VON DER HEIDE, Rebeca; VYAS, Govinda; OLSON, Ingrid. The social network-network: size is predicted by brain structure and function in the amygdala and paralimbic regions. Social cognitive and affective neuroscience, Los Angeles, v. 9, n. 12, p. 1962-72, 2014.

WATERS, Lea et al. Contemplative education: a systematic, evidence-based review of the effect of meditation interventions in schools. Educational Psychology Review, Dordrecht, v. 27, p. 103-134, 2014.

WEARE, Katherine. Mindfulness and contemplative approaches in education. Current Opinion in Psychology, United Kingdom, v. 28, p. 321-326, 2019. 
WITTMANN, Marc; SCHMIDT, Stefan. Mindfulness meditation and the experience of time. studies in neuroscience. In: SCHMIDT, Stefan; WALACH, Harald. Meditation: neuroscientific approaches and philosophical implications. New York: Springer, 2014. p. 199-211.

WU, Jinting; WENNING, Mario. The postsecular turn in education: lessons from the mindfulness movement and the revival of confucian academies. Studies in Philosophy and Education, Netherlands, v. 35, n. 6, p. 551-571, 2016.

ZAJONC, Arthur. Contemplative pedagogy: a quiet revolution in higher education. New Directions for Teaching and Learning, United Kingdom, v. 134, p. 83-94, 2013.

ZENNER, Charlotte; HERRNLEBEN, Solveig; WALACH, Harald. Mindfulness-based interventions in schools: a systematic review and meta-analysis. Frontiers in Psychology, Lausanne, v. 5, n. 603, p. 1-20, 2014.

ZERMEÑO, Ana María; LOZANO-Rodríguez, Armando. Desarrollo de competencias interpersonales en ambientes virtuales. CPU-e Revista de Investigación Educativa, Veracruz, v. 22, p. 176-199, 2016.

Recibido en: 29.05.2019 Aprobado en: 24.03.2020

Francisco José Lería Dulčić es profesor asistente, magíster en Psicología clínica, ph cand. en Lenguaje y neurociencia cognitiva, post-diplomado en Psicoterapia de integración. Líneas de investigación: Psicolingüística de lenguaje afectivo en los contextos educacionales; Orientación contemplativa en la enseñanza y el aprendizaje; Formación inicial docente. 